\title{
Should we be using safety syringes?
}

\author{
Introducing safety syringes into a UK dental school — a controlled study by J. M. Zakrzewska, I. Greenwood, and \\ J. Jackson BrDent J 2001; 190: 88-92
}

\begin{abstract}
Aim
How an appropriate safety syringe was chosen, how the changeover to it was achieved and what outcome measures were used to measure the effectiveness of this change.
\end{abstract}

\section{Introduction}

One third of all reported sharps injuries in dental practice are due to the use of non disposable dental syringes with most injuries being sustained during removal and disposal of the disposable needle from the non-disposable syringe.

\section{Method}

After evaluation of all available disposable safety syringes they were introduced into a dental school after appropriate education of all staff and students. Risk management provided data on all reported needle-stick injuries in the dental school and a control unit using non disposable syringes for a period of two years.

\section{Results}

Avoidable needle stick injuries reduced from an average of 11.8 to 0 injuries per 1,000,000 hours worked per year as compared with a control unit who reduced their frequency from 26 to 20 injuries per $1,000,000$ hours worked. The cost of safety syringes is comparable to non-disposable syringes but the reduction in cost of management of needle stick injuries including the psychological effects are significant.

\section{Conclusion}

Education plays a vitally important role in the effective implementation of the change to safety syringes which is advocated for all dentists.

\section{Comment}

Drobably the highest risk of cross infecP tion in the dental surgery is when there is blood to blood contact. This can occur when dental personnel sustain sharps injuries. There are two main times when sharps injuries occur; these are when contaminated instruments are cleaned and when needles are resheathed. Needle resheathing and reuse only occurs in dentistry. Resheathing does present a serious risk of sharps injuries, particularly when inexperienced persons are doing the procedure. In dental hospitals there are a large number of inexperienced and partially experienced students and therefore the risk of sharps injuries is potentially high.

One method of preventing sharps injuries in dental hospitals is to use devices, which shield the operator from the risk of a sharps injury. A number of devices are now available for this purpose. The team at the St Bartholomhew's and the Royal London have carefully evaluated the use of safety syringes which have devices which help prevent sharp's injuries. Before considering the use of safety syringes they did an analysis of sharp's injuries and decided it was worth introducing the devices.

In these cost-conscious days the need for the devices had to be proved through evidence. This team collected the evidence by finding the number of reported sharp's injuries. They then tested four syringes before selecting one make which suited their clinical practice.

Converting any hospital personnel to change instrumentation can be a difficult task, as in hospitals many resist change. The team report in this paper how they set about convincing people of the need for change and how they provided training and support. The need for safe disposal of the instruments and adequate supplies was not overlooked.
Finally, the team evaluated the results of the introduction of the safety syringes. The number of injuries fell to zero in the unit using the safety syringes, which is very impressive, although the period of evaluation was short. The team had thus achieved their aim of introducing the safety syringes and showing that they were not just cost effective but prevented sharp's injuries.

This paper is a careful and detailed evaluation of how to introduce change in hospitals through audit and prospective research. It provides a model that could be used in other situations where change of instrumentation is contemplated. It also provides a blueprint for the presentation of 'case of need' that so many NHS managers seem to need.

\section{V. Martin}

Senior Lecturer and Honorary Consultant Oral Microbiology, University of Liverpool 